\title{
Parasitos gastrointestinais em Puma concolor, Puma yagouaroundi e Leopardus pardalis (Carnivora: Felidae) na Floresta Nacional de Três Barras, SC, Brasil
}

\author{
Gastrointestinal parasites in Puma concolor, Puma yagouaroundi and \\ Leopardus pardalis (Carnivora: Felidae) at the National Forest of \\ Três Barras, SC, Brazil
}

\author{
Derlise Maria Wrublewski ${ }^{{ }^{*}}$, Suellen Cristine Kusma ${ }^{1}$, Valéria Natascha Teixeira ${ }^{2}$ \\ ${ }^{1}$ Universidade Estadual do Paraná (UNESPAR), União da Vitória, PR, Brasil \\ 2 Pontifícia Universidade Católica do Paraná (PUCPR), Curitiba, PR, Brasil
}

\section{Resumo}

Parasitos ocorrem praticamente em todos os níveis tróficos e sua transmissão geralmente depende da presença de hospedeiros intermediários, paratênicos e definitivos dentro de um ecossistema. Entretanto, altas densidades de parasitos nos indivíduos podem expandir os danos em populações e ocasionar endemias. 0 presente estudo analisou a fauna parasitária a partir de exames coproparasitológicos de Puma concolor, Puma yagouaroundi e Leopardus pardalis, de material coletado em trilhas da Floresta Nacional de Três Barras, Santa Catarina, Brasil. Foram realizadas 10 coletas entre os meses de agosto de 2013 e fevereiro de 2014, e as espécies de felídeos foram identificadas através da análise do padrão microestrutural dos pêlos-guarda nas amostras fecais. Os exames coproparasitológicos foram realizados em 45 amostras pelos métodos de Willis e Mollay e Hoffmann, Pons e Janer. Todas as amostras fecais estavam positivas para parasitos, demonstrando a presença de protozoários, nematódeos, trematódeos, cestódeos e ácaros. Foram identificadas 22 formas parasitárias em amostras de L. pardalis, sete em P. concolor e oito em P. yagouaroundi. Alguns parasitos identificados são comumente observados em felídeos, outros são provenientes da fauna parasitária das presas ingeridas, além de ovos de parasitos comuns em animais domésticos, demonstrando a presença da ação antrópica na área de estudo, o que pode ser considerado um agravante à situação de risco desses felídeos ameaçados de extinção.

Palavras-chave: Parasitologia. Felídeos silvestres. Coproparasitológico. Tricologia. Floresta Nacional.

\begin{abstract}
Parasites occur in practically all trophic levels and their transmission can usually depend on the presence of intermediaries, paratenic and definitive hosts in an ecosystem. However, high densities of parasites in the
\end{abstract}


individuals can expand the damage in populations and cause epidemics. This study analyzed the parasitic fauna from Puma concolor, Puma yagouaroundi and Leopardus pardalis, in fecal samples collected on the trails of the National Forest of Três Barras, Santa Catarina, Brazil. There were 10 collections carried out between the months of August 2013 to February 2014. The species of felids were identified through microstructural pattern analysis of hair-guard in the fecal samples. The fecal examinations were performed on 45 samples with the methods of Willis and Mollay and Hoffmann, Pons and Janer. All the fecal samples were positive for parasites, demonstrating the presence of protozoa, nematodes, trematodes, cestodes and mites. There were 22 parasitic forms identified in the $\mathrm{L}$. pardalis samples, seven in the P. concolor and eight in the P. yagouaroundi. Some identified parasites are commonly seen in felines, others are from the parasitic fauna of ingested prey, and common parasites eggs of domestic animals, demonstrating the presence of human activities in the study area, what can be considered as an aggravating of the risk situation of these endangered species of felids.

Keywords: Parasitology. Wild felids. Coproparasitological. Trichology. National Forest.

\section{Introdução}

Parasitismo é uma relação interespecífica na qual o hospedeiro beneficia o parasito com alimento e este se mantém vivo pela manutenção do padrão metabólico do seu hospedeiro (Odum, 2010). Nem sempre o parasitismo caracteriza uma doença, entretanto, há casos em que esta relação causa danos aos hospedeiros, acarretando em déficits metabólicos geralmente causados pela elevada densidade de parasitos e/ou por parasitos que desencadeiam doenças com as quais o sistema imunológico do hospedeiro não está habituado (Neves, 2003).

Altas densidades de parasitos nos indivíduos podem expandir os danos em populações e ocasionar endemias (Rapport et al., 1998). Por isso, as análises da biodiversidade de parasitos em animais silvestres em áreas de conservação podem ser importantes indicadores de saúde destes ecossistemas, refletindo a filogenia, as condições parasito-hospedeiro e as pressões evolutivas sobre ambos no ambiente (Lymbery, 2005), além de possibilitar a observação de fluxos migratórios e de dispersão, elos de cadeias tróficas e alterações de dieta, hábitos e comportamentos de hospedeiros (Bongers e Ferris, 1999; Wood, 2006).

As doenças parasitárias são um dos principais indicadores de desequilíbrio dos ecossistemas e acarretam em aumento dos riscos para as populações que os compõem (Rapport etal., 1998), visto que a alta vulnerabilidade desses animais à contaminação por parasitos debilitantes ou letais (Murray et al., 1999) pode ter consequências maiores em populações que já são consideradas ameaçadas (O'Brien et al., 1985). Nos diversos estados brasileiros, a maioria dos felídeos silvestres está classificada sob algum grau de ameaça e algumas espécies apresentam grave perigo de extinção (Reis et al., 2011).

Os parasitos ocorrem em quase todos os níveis tróficos e sua transmissão nos felídeos geralmente está associada à contaminação oral, como ingestão de água contaminada e de hospedeiros intermediários ou paratênicos, penetração ativa e até mesmo na disseminação por pessoas que circulam na área (Anderson, 2000; Dryden, 1996). Variações interespecífica e intraespecífica de infecções por parasitos também podem estar correlacionadas com o ambiente, demografia, comportamento e alterações ambientais causadas pela ação antrópica (Stuart e Strier, 1995).

Vários autores descrevem a presença de parasitos em felídeos selvagens (Patton et al., 1986; Rickard e Foreyt, 1992; Patton e Rabinowitz, 1994; Xavier et al., 2000; Freitas et al., 2001; Pence et al., 2003; Tantaleán e Michaud, 2005; Vieira et al., 2008; Santos et al., 2009), porém, apesar da importância do ponto de vista conservacionista, são poucos os dados recentes. Estudos de parasitos em felídeos ameaçados no sul do Brasil ainda são incipientes, principalmente nos animais que estão em áreas protegidas. Sendo assim, este é o primeiro estudo desse tipo na Floresta Nacional de Três Barras (Flona), e teve por objetivo identificar as formas parasitárias de três espécies de felídeos silvestres de vida livre - Puma concolor (Linnaeus, 1771), Puma yagouaroundi (E. Geoffroy SaintHilare, 1803) e Leopardus pardalis (Linnaeus, 1758) - por meio de exames coproparasitológicos de amostras encontradas sobre o solo de trilhas, 
contribuindo com os estudos da fauna parasitológica gastrointestinal dessas espécies e oferecendo informações sobre a interação entre parasitos, presas e predadores e as condições ambientais dentro deste ecossistema.

\section{Materiais e Métodos}

\section{Área de estudo}

O estudo foi realizado na Flona (Figura 1), cuja área é de 4.458,5 hectares, localizada no município

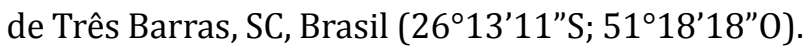

Trata-se de uma Unidade de Uso Sustentável administrada pelo Instituto Chico Mendes de Conservação da Biodiversidade e inserida no Bioma Mata Atlântica sob a Floresta Ombrófila Mista, também conhecida como Mata das Araucárias. A área tem como corte na ponta norte de sua extensão a BR-280 (Marques, 2007).

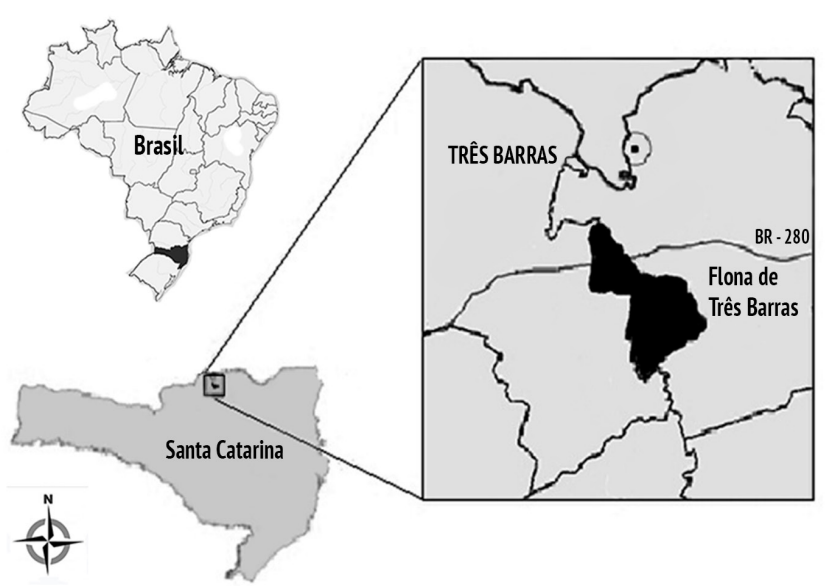

Figura 1 - Mapa demonstrativo da região onde está inserida a Floresta Nacional de Três Barras (adaptado de Marques, 2007).

\section{Coleta de material fecal}

Entre agosto de 2013 e fevereiro de 2014, foram empreendidas, quinzenalmente, caminhadas à procura de amostras fecais sobre estradas já existentes, em um trajeto de 13 quilômetros, enquadrando áreas de vegetação nativa, reflorestamento de araucária, reflorestamento de pinus e uma área de maior antropização, localizada próxima à sede da Flona. Foram realizados 10 episódios de coleta do material fecal, totalizando um esforço de campo estimado em aproximadamente 80 horas e 130 quilômetros percorridos.

Cada amostra foi dividida em duas parcelas e armazenada em refrigeração, sendo a primeira utilizada para identificação da espécie de hospedeiro e a segunda para o exame coproparasitológico. 0 procedimento adotado na identificação da espécie hospedeira foi realizado em laboratório por meio da identificação microscópica de pêlos-guarda, pela observação dos padrões microestruturais medulares e cuticulares (Quadros, 2002; Quadros e Monteiro-Filho, 2006a, 2006b).

\section{Análise coproparasitológica}

As amostras destinadas ao exame coproparasitológico foram mantidas em caixas térmicas refrigeradas e analisadas pelos métodos de Willis e Mollay (1921) e de Hoffmann, Pons e Janer (1934). Os procedimentos de identificação dos parasitos encontrados pelos métodos foram feitos por meio de observação das formas parasitárias em microscópio óptico (Zajac e Conboy, 2012).

\section{Análise de dados}

0 registro dos parasitos encontrados nas espécies P. concolor, P. yagouaroundi e L. pardalis foi feito considerando-se todas as espécies de parasitos encontradas, mesmo as com baixa abundância relativa.

\section{Resultados}

Foram coletadas no total 45 amostras fecais, sendo identificadas as três espécies de interesse, quatro de Puma concolor, 10 de Puma yagouaroundi e 31 de Leopardus pardalis. Não foi observada relação entre as estações do ano e a quantidade de fezes coletadas durante este período.

Todas as amostras de Puma concolor analisadas apresentaram formas parasitárias, sendo uma amostra negativa apenas no método de 
sedimentação de Hoffmann. Foram identificados parasitos dos filos Protozoa, Platyhelminthes (das classes Cestoda e Trematoda), Nematoda e Arthropoda (da classe Arachnida) (Tabela 1).

Todas as amostras de Puma yagouaroundi foram positivas para formas parasitárias, sendo identificados parasitos dos filos Platyhelminthes (das classes Cestoda e Trematoda), Nematoda e Arthropoda (da classe Arachnida).

Todas as amostras de fezes pertencentes a Leopardus pardalis apresentaram formas parasitárias pertencentes aos filos Protozoa, Platyhelminthes (das classes Cestoda e Trematoda), Nematoda e Arthropoda (da classe Arachnida).

Tabela 1 - Frequência de formas parasitárias encontradas nas amostras fecais de Leopardus pardalis, Puma concolor e Puma yagouarondi provenientes da Floresta Nacional de Três Barras, SC

\begin{tabular}{|c|c|c|c|c|c|c|}
\hline \multirow{2}{*}{ Forma parasitária } & \multicolumn{2}{|c|}{ L. pardalis $(n=31)$} & \multicolumn{2}{|c|}{ P. concolor $(n=4)$} & \multicolumn{2}{|c|}{ P. yagouaroundi $(\mathrm{n}=10)$} \\
\hline & NP & $\mathbf{F}$ & NP & $\mathbf{F}$ & NP & $\mathbf{F}$ \\
\hline \multicolumn{7}{|l|}{ Filo Nematoda } \\
\hline Adulto de Oxyuridae & 1 & $3,2 \%$ & - & - & - & - \\
\hline Adultos de Strongyloides sp. ${ }^{*}$ & 1 & $3,2 \%$ & - & - & - & - \\
\hline Larva de Aelurostrongylus sp. & 10 & $31,0 \%$ & - & - & 2 & $20,0 \%$ \\
\hline Ovos de Ancylostomatidae & 8 & $25,0 \%$ & 1 & $25,0 \%$ & 2 & $20,0 \%$ \\
\hline Ovos de Ascaroidea & 2 & $6,4 \%$ & - & - & 1 & $10,0 \%$ \\
\hline Ovos de Capillaria sp. & 7 & $22,5 \%$ & - & - & 3 & $30,0 \%$ \\
\hline Ovos de Oxyuris equi & 1 & $3,2 \%$ & - & - & - & - \\
\hline Ovos de Passalurus sp. & 1 & $3,2 \%$ & - & - & - & - \\
\hline Ovos de Spiruridae & 1 & $3,2 \%$ & 1 & $25,0 \%$ & - & - \\
\hline Ovos de Strongyloides sp. & 1 & $3,2 \%$ & - & - & - & - \\
\hline Ovos de Toxocara sp. & 6 & $19,3 \%$ & 1 & $25,0 \%$ & - & - \\
\hline Ovos de Trichuris sp. & 4 & $12,9 \%$ & - & - & - & - \\
\hline \multicolumn{7}{|l|}{ Classe Cestoda } \\
\hline Ovos de Cestoda & 1 & $3,2 \%$ & - & - & - & - \\
\hline Ovos de Spirometra sp. & 21 & $67,7 \%$ & 3 & $75,0 \%$ & 7 & $70,0 \%$ \\
\hline \multicolumn{7}{|l|}{ Classe Trematoda } \\
\hline Ovos de Alaria sp. & 3 & $9,6 \%$ & 1 & $25,0 \%$ & - & - \\
\hline Ovos de Paragonimus sp. & 6 & $19,3 \%$ & - & - & 3 & $30,0 \%$ \\
\hline \multicolumn{7}{|l|}{ Filo Protozoa } \\
\hline Oocistos de Coccidia & 1 & $3,2 \%$ & - & - & - & \\
\hline Oocistos de Eimeria sp. & 3 & $9,6 \%$ & 1 & $25,0 \%$ & - & - \\
\hline Oocistos de Sarcocystis sp. & 2 & $6,4 \%$ & - & - & - & - \\
\hline \multicolumn{7}{|l|}{ Classe Arachnida } \\
\hline Adulto de Acari & 3 & $9,6 \%$ & 2 & $50,0 \%$ & - & - \\
\hline Adulto de Demodex sp. & 1 & $3,2 \%$ & - & - & 1 & $10,0 \%$ \\
\hline Ovos de Acari & 9 & $29,0 \%$ & - & - & 5 & $50,0 \%$ \\
\hline
\end{tabular}

Nota: $\mathrm{n}$ = número de amostras coletadas; $\mathrm{NP}$ = número de amostras positivas para a forma parasitária; $\mathrm{F}=$ Frequência da forma parasitária na espécie (\%) * Nematóides machos e fêmeas. 
Foram identificadas 22 formas parasitárias nas amostras fecais de L. pardalis, sendo elas: adultos de Strongyloides sp.; larvas de Aelurostrongylus sp., ovos de Ancylostomatidae, Ascaroidea, Capillaria sp., Passalurus sp., Spiruridae, Strongyloides sp., Toxocara sp., Trichuris sp., ovos de Cestoda, Spirometra sp., Alaria sp. e Paragonimus sp.; oocistos de Coccidia, Eimeria sp. e Sarcocystis sp.; adulto de Demodex sp., ovos de Acari e de Oxyuris equi (Figura 2).

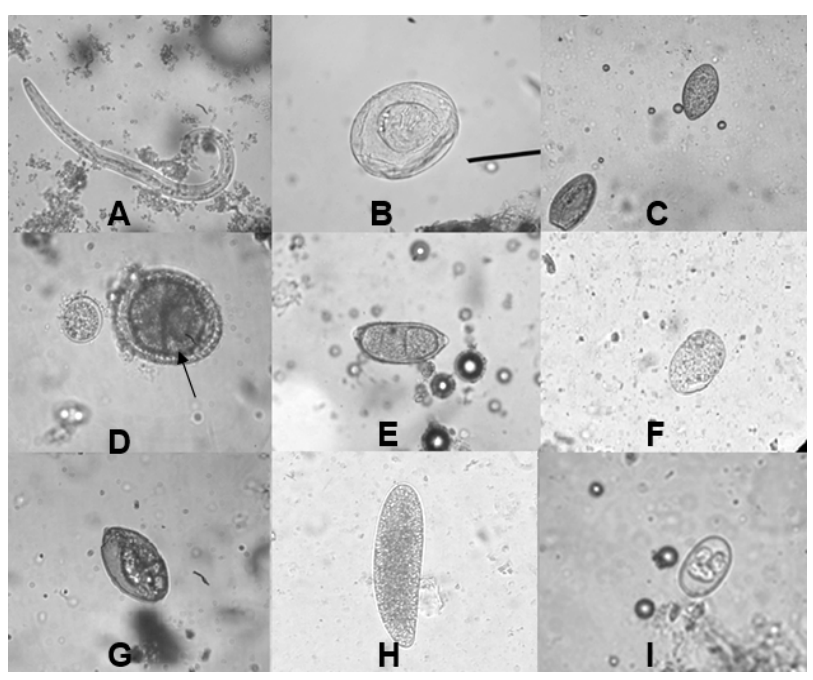

Figura 2 - A) Larva de Aelurostrongylus sp. B) Ovo de (estoda. C) Ovos de Spirometra sp. D) Ovo de Toxocara sp. (seta) e oocisto. E) Ovo de Capillaria sp. F) Ovo de Ancylostomatidae. G) Ovo de Paragonimus sp. H) Ovo de Acari. I) Oocisto de Eimeria sp.

Nas amostras de $P$. concolor foram identificados ovos de Ancylostomatidae, Spiruridae, Toxocara sp., Spirometra sp. e Alaria sp., e oocistos de Eimeria sp. e Acari adulto.

As formas parasitárias encontradas nas amostras de P. yagouaroundi foram larva de Aelurostrongylus sp.; ovos de Ancylostomatidae, Ascaroidea, Capillaria sp., Paragonimus sp., Spirometra sp. e Acari e adulto de Demodex sp.

\section{Discussão}

Todas as amostras fecais foram positivas para formas parasitárias, assim como outros trabalhos com felídeos de vida livre que relatam uma frequência que varia de $86,7 \%$ (Patton et al., 1986) a 100\% (Rickard e Foreyt, 1992; Patton e Rabinowitz, 1994; Pence et al., 2003) de amostras parasitadas.

Parasitos dos gêneros Spirometra, Strongyloides, Toxocara, entre outros foram relatados em felídeos de cativeiro e de vida livre por vários autores (Patton et al., 1986; Xavier et al., 2000; Freitas et al., 2001; Tantaleán e Michaud, 2005; Vieira et al., 2008), porém, diferente do presente estudo, nenhum dos trabalhos encontrados na literatura utilizou a tricologia como forma de identificação da espécie autora das fezes, descrevendo os parasitos gastrointestinais de felídeos de vida livre em áreas relativamente grandes sem que houvesse captura dos felídeos ou teste genético das fezes.

Alguns parasitos identificados são comumente observados em felídeos, possuem ciclo direto e não causam doenças graves, como é o caso de Ancylostomatidae, Ascaroidea e Trichuris sp. (Chame, 1988; Duarte, 1993; Noronha et al., 2002; Beldomenico et al., 2005). Já Paragonimus sp., Spiruridae, Spirometra sp., Aelurostrongylus sp., Alaria sp. e Capillaria sp. podem ser adquiridos pela ingestão de presas que atuam como hospedeiros intermediários ou paratênicos, como répteis, aves e pequenos mamíferos (Balmori et al., 2000; Hidalgo, 2005; Bowman et al., 2006; Zajac e Conboy, 2006; Monteiro, 2011; Zajac e Conboy, 2012).

Outros parasitos identificados são provenientes da ingestão de hospedeiros definitivos, como é o caso de Passalurus sp., Capillaria sp., oocistos de protozoários, entre outros comuns em roedores, lebres e aves (Rodriguez et al, 1973; Pessoa, 1988; Monteiro, 2011), o que sugere que o seu diagnóstico no presente estudo pode ser explicado pela interação presa-parasito-predador, tendo os felídeos o papel de transporte e disseminação dos ovos no ambiente.

Também foram identificados em $P$. concolor, $P$. yagouaroundi e L. pardalis ácaros e ovos de ácaros, que são ectoparasitos comuns em folículos pilosos de várias espécies de mamíferos (Yager e Scott, 1985), diagnóstico que pode estar relacionado ao comportamento predador dos felídeos, que acabam eliminando nas fezes os resíduos provenientes da presa. Outra condição que pode justificar a presença de ácaros e ovos de ácaros nas amostras fecais das espécies estudadas é o hábito de autolimpeza dos 
felídeos, que podem ingerir esses ectoparasitos presentes nos próprios pêlos.

Oxyuris equi, identificado em uma amostra de $L$. pardalis, é comum no intestino grosso de equinos (Anderson, 1992). Esse achado pode ser resultante de contaminação ambiental, tendo L. pardalis ingerido os ovos através do solo ou da água e os disseminado através de suas fezes. Adicionalmente, também foram encontrados protozoários comuns do ceco de cavalos, reafirmando a possibilidade de ingestão acidental de resíduos fecais equinos em água ou pelo comportamento de autolimpeza do felídeo.

Strongyloides sp. é descrito principalmente em gatos domésticos em vários países do mundo, inclusive no Brasil (Vicente et al., 1997; Bowman et al., 2002), tendo um único registro em felídeos silvestres no país na espécie L. tigrinus (Santos et al., 2009). Machos adultos de Strongyloides sp. são nematoides de vida livre, não estando relacionados diretamente com a infecção dos felídeos. 0 achado desta forma parasitária também pode estar relacionado à ingestão destes nematoides em água contaminada ou ao hábito de autolimpeza dos felídeos. Apesar de ser uma Unidade de Conservação, a Flona sofre influência da antropização do entorno, podendo levar à interação de felídeos selvagens com gatos domésticos. Para Beldomenico et al. (2005), a infecção por Toxocara sp. em felídeos silvestres também pode estar relacionada à interação com gatos domésticos.

As parasitoses gastrintestinais e respiratórias em felídeos, de modo geral, podem acarretar em anemia, emagrecimento, menor capacidade de se adaptar às mudanças do ambiente e doenças como inflamação, obstrução intestinal, bronquite crônica e pneumonia (Monteiro, 2011; Aranda et al., 2013).

Os resultados do presente trabalho ressaltam a importância de manter os ambientes preservados para garantir a saúde ambiental das populações que dividem a área, principalmente as que envolvem esforço conservacionista por causa da situação de risco, como é o caso de L. pardalis, $P$. concolor e $P$. yagouarondi, consideradas espécies ameaçadas de extinção na categoria "vulnerável" no Brasil (Chiarello et al., 2008; Brasil, 2014), e L. pardalis e $P$. concolor, categorizadas em Santa Catarina como "em perigo" e "vulnerável", respectivamente (Santa Catarina, 2011). Além dos vários fatores que as ameaçam, como a caça, a destruição e a fragmentação de habitats (Oliveira e Cassaro, 2005; Chiarello et al., 2008; Reis et al., 2011), a contaminação ambiental e a transmissão de parasitos por animais domésticos pode prejudicar seus ciclos de vida e contribuir para a redução de suas populações. Ambientes antropizados são seletivos para populações generalistas que se adaptam a condições de exploração da área e podem reduzir a biodiversidade, promover competição e até ameaçar as populações mais sensíveis (Mangini et al., 2012).

\section{Conclusão}

Os dados aqui apresentados trazem importantes contribuições à parasitologia veterinária e à ecologia dos felídeos selvagens estudados, sendo que muitos achados corroboram com o descrito na literatura e outros permitem observar a dinâmica da relação entre parasitos, presas e predadores. Foi possível observar ovos de parasitos que dão indícios de contaminação ambiental na área de estudo por fezes de animais domésticos, o que pode ser considerada uma grave ameaça aos felídeos em risco de extinção.

\section{Referências}

Anderson RC. Nematode parasites of vertebrates: their development and transmission. Farnham: CAB International; 1992. p. 214-5.

Anderson RC. Nematode parasites of vertebrates: their development and transmission. 2 ed. Wallingford: CAB Publishing; 2000.672 p.

Aranda C, Serrano-Martínez E, Tentaléan M, Quispe M, Casas G. Identificación y frecuencia de parásitos gastrointestinales en félidos silvestres en cautiverio en el Perú. Rev Inv Vet Perú. 2013;24(3):360-8.

Balmori A, Rico M, Naves J, Llamazares E. Contribución al estudio de los endoparásitos del lobo en la Península Ibérica: una investigación coprológica. Galemys. 2000;12(n. esp):13-26. 
Beldomenico PM, Kinsella JM, Uhart MM, Gutierrez L, Pereira J. Helminths of Geoffroy's cat, Oncifelis geoffroyi (Carnivora, Felidae) from the Monte desert, central Argentina. Acta Parasitol. 2005;50(3):263-6.

Bongers T, Ferris H. Nematode community structure as a bioindicator in environmental monitoring. Trends Ecol Evol. 1999;14(6):224-8.

Bowman DD, Hendrix CM, Lindsay DS, Barr SC. Feline Clinical Parasitology. Ames: Iowa State University Press; 2002. p. 469.

Bowman DD, Lynn RC, Eberhard ML, Alcaraz A. Parasitologia Veterinária de Georgis. São Paulo: Manole; 2006.

Brasil. Portaria no 444, de 17 de dezembro de 2014. Lista Nacional Oficial de espécies ameaçadas de extinção [acesso 29 jan 2015]. Disponível em: https://tinyurl. com/ycg27q9r.

Chame M. Estudo comparativo das fezes e coprólitos não humanos da região arqueológica de São Raimundo Nonato, Sudeste do Piauí [dissertação]. Rio de Janeiro: Universidade Federal do Rio de Janeiro; 1988.

Chiarello AG, Aguiar LMS, Cerqueira R, Melo FR, Rodrigues FHG, Silva VMF. Mamíferos Ameaçados de Extinção no Brasil. In: Machado ABM, Drummond GM, Paglia AP (EE). Livro Vermelho da Fauna Brasileira Ameaçada de Extinção. Brasília: Fundação Biodiversitas; 2008. p. 681-880.

Dryden MW. Diagnosis and control of gastrointestinal parasites in dogs and cats. Vet Q. 1996;18(supl. 1):42-3.

Duarte AN. Estudo paleoparasitológico em coprólitos do sítio arqueológico da furna do estrago, município do Brejo da Madre de Deus, Pernambuco [dissertação]. Rio de Janeiro: Universidade Federal Rural do Rio de Janeiro; 1993.

Freitas MFL, Oliveira JB, Cavalcanti MDB, Oliveira RA, Sobrinho AE. Perfil coproparasitológico de mamíferos silvestres en cautiverio en el estado de Pernambuco, Brasil. Parasitol Día. 2001;25(3-4):121-5.
Hidalgo MEC. Paragonimíase. In: Coura JR. Dinâmica das doenças infecciosas e oarasitárias. 1 ed. Rio de Janeiro: Guanabara Koogan; 2005.

Hoffman WA, Pons JA, Janer JL. The sedimentationconcentration method in Schistosomiasis mansoni. PR J Public Health Trop Med. 1934;9:281-98.

Lymbery AJ. Parasite an ecosystem health. Int J Parasitol. 2005;35(2005):703.

Mangini PR, Jorge RSP, Santos MRD, Filoni C, Verona CES, Nava A, et al. Conservation Medicine in Brazil: case studies of ecological health in preactice. In: Aguirre AA, Ostfeld RS, Daszak P (EE). New directions in conservation medicine: applied cases of ecological health. New York: Oxford University Press; 2012.

Marques AC. Planejamento da paisagem da Floresta nacional de Três Barras (Três Barras-SC): subsídios ao plano de manejo [dissertação]. Curitiba: Universidade Federal do Paraná; 2007.

Monteiro SG. Parasitologia na Medicina Veterinária. São Paulo: Roca; 2011.

Murray DL, Kapke CA, Evermann JF, Fuller TK. Infectious disease and the conservation of free-ranging large carnivores. Anim Conserv. 1999;2(4):241-54.

Neves DP. Parasitologia Humana. 10 ed. São Paulo: Atheneu; 2003.

Noronha D, Vicente JJ, Pinto RM. A survey of new records for nematodes from mammals deposited in the Helminthological collection of the Institute Oswaldo Cruz (CHIOC). Rev Bras Zool. 2002;19(3):945-9.

O'brien S, Roelke ME, Marker L, Newman A, Winkler CA, Meltzer D, et al. Genetic basis for species vulnerability in the cheetah. Science. 1985;227(4693):1428-34.

Odum EP. Ecologia. Rio de Janeiro: Guanabara Koogan; 2010.

Oliveira TG, Cassaro K. Guia de campo dos felinos do Brasil. São Paulo: Instituto Pró-Carnívoros, Sociedade de 
Zoológicos do Brasil, Fundação Parque Zoológico de São Paulo; 2005. 80 p.

Patton S, Rabinowitz A, Randolph S, Johnson SS. A coprological survey of parasites of wild neotropical felidae. J Parasitol. 1986;72(4):517-20.

Patton S, Rabinowitz AR. Parasites of wild felidae in Thailand: a coprological survey. J Wildl Dis. 1994;30(3):472-5.

Pence DB, Tewes ME, Laack LL. Helminths of the ocelots of southern Texas. J Wildl Dis. 2003;39(3):683- 9.

Pessoa SB. Parasitologia Médica. Rio de Janeiro: Guanabara Koogan; 1988.

Quadros J. Identificação microscópica de pêlos de mamíferos brasileiros e sua aplicação no estudo da dieta de carnívoros [tese]. Curitiba: Universidade Federal do Paraná; 2002.

Quadros J, Monteiro-Filho ELA. Revisão conceitual, padrões microestruturais e proposta nomenclatória para os pêlos-guarda de mamíferos brasileiros. Rev Bras Zool. 2006a;23(1):279-92.

Quadros J, Monteiro-Filho ELA. Coleta e preparação de pêlos de mamíferos para identificação em microscopia óptica. Rev Bras Zool. 2006b;23(1):274-8.

Rapport D, Costanza R, Epstein P, Gaudet C, Levins, R. Ecosystem Health. Malden, MA: Blackwell Science; 1998.

Reis NR, Peracchi AL, Pedro WA, Lima IP. Mamíferos do Brasil. 2 ed. Londrina: Edição do Autor; 2011.

Rodriguez JR, Pozo DG, Herrera JL. Estudios sobre el genero Passalurus, Dujardin, 1845, parasitando al Oryctolagus cuniculus domestica (L.) y Lepus granatensis (R.). Rev Iber Parasitol. 1973;33(2-3):315-29.

Rickard LG, Foreyt WJ. Gastrointestinal parasites of cougars (Felis concolor) in Washington and the first report of Ollulanus tricuspis in a sylvatic felid from North America. J Wildl Dis. 1992;28(1):130-3.

Santa Catarina. Resolução CONSEMA no 002, de 06 de dezembro de 2011. Reconhece a lista oficial de espécies da fauna ameaçadas de extinção no estado de Santa Catarina [acesso 29 jan 2015]. Disponível em: https:// tinyurl.com/y9x593er.

Santos KR, Faciulli P, Paparotto T, Takahira RK, Lopes RS, Silva RJ. First report of Strongyloides sp. (Nematoda, Strongyloididae) in Leopardus tigrinus (Carnivora: Felidae) in the municipality of Botucatu, State of São Paulo, Brazil. Rev Bras Parasitol Vet. 2009;18(supl. 1):77-9.

Stuart MD, Strier KB. Primates and parasites: a case for a multidisciplinary approach. Int J Primatol. 1995;16(4):577-93.

Tantaleán M, Michaud C. Huéspedes definitivos de Spirometra mansonoides (Cestoda, Diphyllobothriidae) en Perú. Rev Peru Biol. 2005;12(1):153-7.

Vicente JJ, Rodrigues HO, Gomes DC, Pinto RM. Nematóides do Brasil. Parte V: Nematóides de mamíferos. Rev Bras Zool. 1997;14(supl. 1):1-452.

Vieira FM, Luque JL, Muniz-Pereira LC. Checklist of helminths parasites in wild carnivore mammals from Brazil. Zootaxa. 2008;1721:1-23.

Willis HH. A simple levitation method for the detection of hookworm ova. Med J Aust. 1921;2(18):375-6.

Wood MJ. Parasites entangled in food webs. Trends Parasitol. 2006;23(1):8-10.

Xavier F, Joseph GK, Michael B, Bijula K. A coprological survey of parasites of seven mammal groups atSilent Valley National Park, Kerala. Zoos Print J. 2000;15(6):279-80.

Yager JA, Scott DW. The skin and appendages. In: Jubb KVF, Kennedy PC, Palmer N (EE). Patology of Domestic Animals, vol. 1. 3 ed. Orlando: Academic Press; 1985.

Zajac AM, Conboy GA. Fecal examination for the diagnosis of parasitism. In: Zajac AM, Conboy GA. Veterinary Clinical Parasitology. Hoboken: Blackwell Publishing; 2006. p. $52-3$.

Zajac AM, Conboy GA. Veterinary clinical parasitology. 8 ed. Ames: Wiley- Blackwell; 2012. 